\title{
Multi-Relational and Social-Influence Model for Predicting Student Performance in Intelligent Tutoring Systems (ITS)
}

\author{
Kouamé Abel Assielou, Cissé Théodore Haba, Tanon Lambert Kadjo, \\ Kouakou Daniel Yao, Bi Tra Goore
}

\begin{abstract}
Recent studies have shown that Matrix Factorization (MF) method, deriving from recommendation systems, can predict student performance as part of Intelligent Tutoring Systems (ITS). In order to improve the accuracy of this method, we hypothesize that taking into account the mutual influence effect in the relations of student groups would be a major asset. This criterion, coupled with those of the different relationships between the students, the tasks and the skills, would thus be essential elements for a better performance prediction in order to make personalized recommendations in the ITS. This paper proposes an approach for Predicting Student Performance (PSP) that integrates not only friendship relationships such as workgroup relationships, but also mutual influence values into the Weighted Multi-Relational Matrix Factorization method. By applying the Root Mean Squared Error (RMSE) metric to our model, experimental results from KDD Challenge 2010 database show that this approach allows to refine student performance prediction accuracy.
\end{abstract}

Keywords : Matrix Factorization, Student Performance Prediction, Intelligent Tutoring System, Social-Influence, Recommender Systems.

\section{INTRODUCTION}

Intelligent Tutoring Systems development began in the 1970s with the goal to improve Computer-Assisted Learning

Revised Manuscript Received on February 05, 2020.

* Correspondence Author

Kouamé Abel Assielou*, Laboratoire de Recherche, Informatique Télécommunication (LARIT), Institut National Polytechnique Felix Houphouet Boigny (INP-HB), Yamoussoukro, Côte d'Ivoire. E-mail:kouame.assielou@inphb.ci

Cissé Théodore Haba, Department of Training and Research of Electrical \& Electronics Engineering, Institut National Polytechnique Felix Houphouet Boigny (INP-HB), Yamoussoukro, Côte d'Ivoire.

E-mail: cissetheodore@yahoo.fr

Tanon Lambert Kadjo, Laboratoire De Recherche en Informatique E Télécommunication (LARIT), Institut National Polytechnique Felix Houphouet Boigny (INP-HB), Yamoussoukro, Côte d'Ivoire.

E-mail: nonatipv6@yahoo.fr

Kouakou Daniel Yao, Laboratory of studies and prevention in Psychoeducation (LEPPE-ENS), University Jean Lorougnon Guédé, Daloa, Côte d'Ivoire. E-mail: yahaudan@gmail.com

Bi Tra Goore, Laboratoire de Recherche en Informatique et Télécommunication (LARIT), Institut National Polytechnique Felix Houphouet Boigny (INP-HB), Yamoussoukro, Côte d'Ivoire.

E-mail: bitra.goore@gmail.com

(c) The Authors. Published by Blue Eyes Intelligence Engineering and Sciences Publication (BEIESP). This is an open access article under the CC BY-NC-ND license (http://creativecommons.org/licenses/by-nc-nd/4.0/)
(CAL). STI-driven progress needed to be operationalized through the use of Artificial Intelligence (AI) methods to provide highly personalized feedback-based education tailored to the needs of the students. Their aim is to support learning by simulating the teaching skills and field expertise of hu-man tutors and to produce the same kind of learning and flexibility between teachers and students [1]. An ITS is made up of three domains: Computer Science, Psychology and Education. Specifically, (i) artificial intelligence explains how to reason about intelligence and therefore about learning, (ii) psychology (cognitive science) explains how people think and learn, and (iii) education is about center on the best way to support teaching / learning [2]. Although, there are different Intelligent Tutorial Systems with different architectures, their basic architecture has four components (modules / models) that are (see Figure 1):

- a Domain-Model that defines the content to be taught;

- a Tutoring Model that defines how to teach;

- a Student-Model that can personalize the learning taking into account this one;

- an Interface-Model that defines the visible means allowing the interrelation be-tween student and the system.

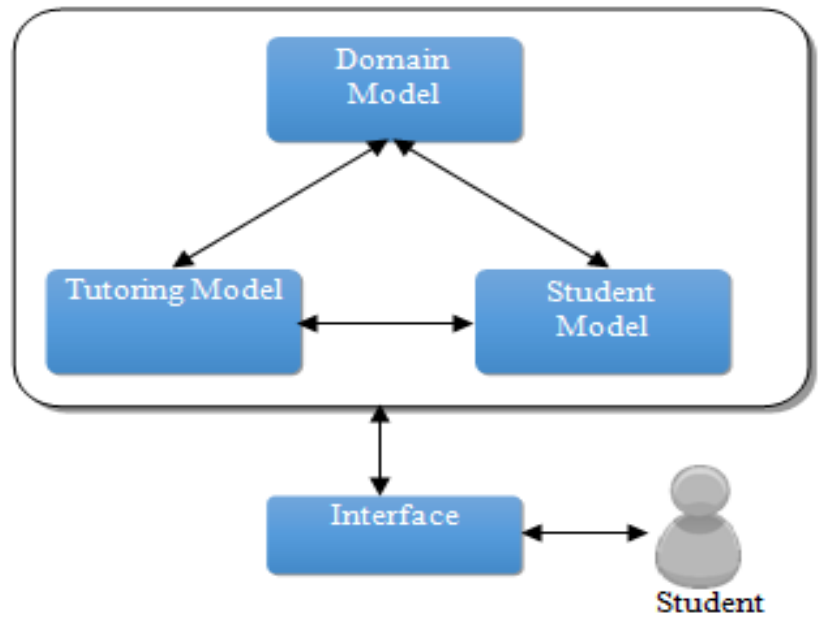

Fig. 1. Classic Intelligent Tutoring System architecture

The Student Model is an important component for more than one reason in any ITS. It can guide the interactions between the student and the system. Student modeling is used to represent student's knowledge evolution, to predict his / her performance for different skills over time [3] and to determine the next content to present to the student to give continuity to his learning [4].

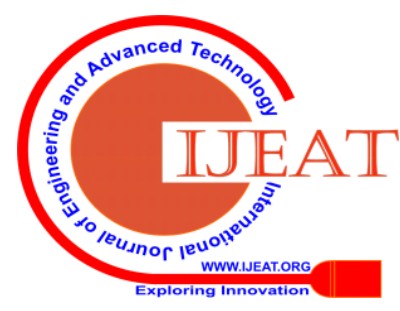


This modeling is also important for re-searchers as it provides them with practical information to make decisions about how to adapt the learning experience to student's needs. Thus, in order to determine the next content to be displayed and specially to adapt the learning, it is important for the current systems, to have a precision of the students' performances prediction. The prediction of student performance is therefore a relevant issue and a major asset for improving learning in all ITS.

In this contribution, we want to solve the PSP problem by proposing a weighted multi-relational matrix factorization model that takes advantage of student-workgroup relationships with the consideration of Student Individual Influence Effect. We seek to provide our model with significant data to improve the accuracy of student performance predicting.

The organization of our paper is as follows: Section 2 provides the state-of-the-art techniques used for predicting student performance. Section 3 presents, in turn, the classical matrix factorization techniques while section 4 describes our multi-relational approach to predict student performance. Section 5 presents an evaluation of this approach and discusses our results with those of the state-of-the-art. Section 6 concludes the paper.

\section{STATE-OF-THE-ART AND RELATED WORK}

Personalized learning has the potential to improve learning process and overall learning outcomes [5][6]. This customization can be achieved through learning content adaptation and the application of an individualized learning strategy. Therefore, it is important for current ITS to have not only relevant information about students but also to predict their performance at each stage of their learning.

In the associated scientific literature, there is a wide variety of work related to Student Performance Predicting. Most of them are based on traditional methods such as Knowledge Tracing (KT) [7], linear regression [8], logistic regression [9], k-NN algorithms [10], support vector machines [11][12] [13][14], decision trees [15][16], neural networks [17][18].

Recently, some authors such as Thai-Nghe and al., [19] [20] proposed to use techniques from the recommendation systems, in this case the Matrix Factorization (MF), for student performance prediction. The PSP problem could be considered as that of the prediction of an evaluation. From this perspective, the student, the task and the performance would become the user, the article and the evaluation respectively as presented in the classical recommendation systems. These researchers showed that the use of this technique could improve prediction results over regression methods by relying on past student performance. Other works like those done in [21] have shown that the MF method can be applied to students' raw scores by taking into account the notes in an interval of 0 and 100 instead of 0 and 1 as the work done in the literature. In [22][23], the authors proposed to improve this method by integrating the temporal effect as the students' knowledge improves over time. Experimental results show that the proposed approaches are promising. However, these works considered only one relation: that existing between the student and the task to be realized. To extend the predictive efficiency of this method, the authors Thai-Nghe and al., [24] proposed to explore multiple relationships that may exist between students, tasks and their metadata using Multi-Relational Matrix Factorization model (MRMF). They also propose a Weighted Multi-Relational Matrix Factorization (WMRMF) model to take into account the main relationship that contains the target variable. The authors Nedungadi et al., [25] propose, in addition to the Multi-Relational factor, to integrate the student's bias, which is defined by the probability that a student performs task and task's bias that can reflect the degree of difficulty of the task. However, these methods have not benefited from social relationships that can be integrated. They ignored significant connections between students (registered in workgroups, for example), which is not in line with real-world observations. It has been shown, in fact, that group work promotes collaboration among students to achieve common learning goals and increases their performance, perseverance, and attitudes [26][27].

With the advent of social networks, social networking approaches to online referral are growing. These approaches, such as those proposed in [28][29][30], assume the existence of a social network among the users to make recommendations to a user on the basis of the evaluations of users having direct or indirect social relations with the given user. In [31], the authors Thanh-Nhan and al., Propose precisely an approach to integrate the social relations of the users / students (for example, a friend of class) in the classic MF. The results show that this approach makes it possible to take advantage of student-to-model relationships and thereby improve forecasting results. However, this approach does not exploit the individual influence factor of group members. It does not exploit the different relationships between students, tasks and their metadata either.

In order to extend the functions of existing systems, Lukasenko [6] aims to define a complete content of a student model that can be taken into account by the intelligent and adaptive functions of knowledge assessment systems and learning. This empirical model contains categories of information such as: contact information, learning style, student current state (mood, mental state, physiological state) current level of knowledge and skills, objectives, learning progress, learning material used, user interface configuration... This point of view is shared by the authors Bicans and al., [32] who propose the automatic addition of students learning style in the computer learning systems in general.

In this paper, we propose MRMF and WMRMF approaches that aggregate not only friendships with integration of the influence effect, but also relationships be-tween students, tasks, and their metadata, so improve the accuracy of PSP in ITS.

\section{MATRIX FACTORIZATION APPROACHES}

\section{A. Classical Matrix Factorization (MF) method}

Let $S$ denote a set of students, $I$ a set of tasks and $P$ a range of possible performance scores. According to the literature of recommendation systems models [33][25], the "student-performs-task" matrix $R$, considering a single attribute, Can be approximated by a product of two small matrices $W_{1}$ (student) and $W_{2}$ (task). Let $R \approx W_{1} W_{2}^{T}$, as illustrated in Figure 2. 
In this relation, $W_{1} \in \mathbb{R}^{S \times F}$ is a matrix where each line $s$ is a vector containing the $F$ latent factors describing the student $s$ and $W_{2} \in \mathbb{R}^{I \times F}$ is a matrix where each line $i$ is a vector containing the $F$ factors latent describing the task $i$. Let $w_{1_{s}}$ and $w_{2_{i}}$ be the respective vectors of the matrices $W_{1}$ and $W_{2}$ such that their elements are designated by $w_{1_{s f}}$ and $w_{2_{i f}}$.
A student $s$ performance for a task $i$ in the framework of MF technique can be predicted by:

$$
\hat{p}_{s i}=\sum_{f=1}^{F} w_{1_{s f}} w_{2_{i f}}=w_{1_{s}} w_{2_{i}}^{T}
$$

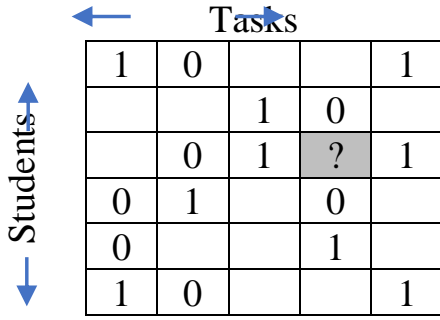

$R$

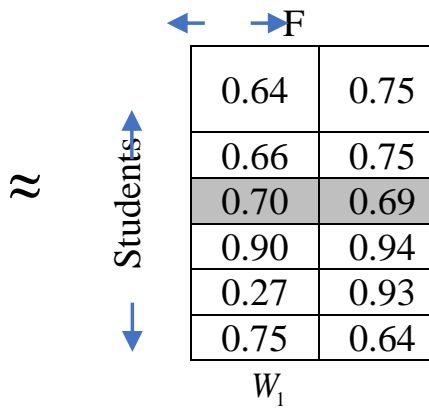

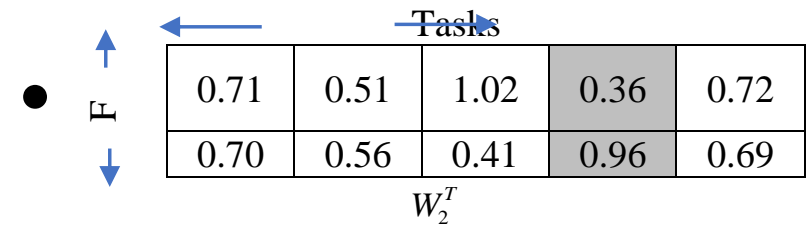

Fig. 2. Example of matrix factorization for the « student-perform-task " relation.

$\hat{p}_{s i}$ is the predicted performance value. $W_{1}$ and $W_{2}$ are the model parameters (latent factor matrices) or factor matrices. These matrices can be learned by optimizing the objective function (2) from a criterion, such as Root Mean Square Error (RMSE), using the stochastic gradient descent method as suggested in [34].

$$
O^{M F}=\sum_{(s, i) \in R}\left(R_{s i}-w_{1_{s}} w_{2_{i}}^{T}\right)^{2}+\lambda\left(\left\|W_{1}\right\|_{F}^{2}+\left\|W_{2}\right\|_{F}^{2}\right)
$$

With $\|\cdot\|_{F}^{2}$ being the Frobenius standard; $\lambda(0 \leq \lambda<1)$ is a regularization term used to avoid over-adjustments. In other words, $\lambda$ is a parameter that make a compromise between the approximation error and the Frobenius norm of the model [33]. Let $e_{s i}$ denote the difference between the real performance value and the predicted performance value for each couple (student, task):

$$
e_{s i}=\left(R_{s i}-w_{1_{s}} w_{2_{i}}^{T}\right)
$$

$R_{s i}$ represents the real value of the student's performance for task $i$.

\section{B. Multi-Relational Matrix Factorization Method (MRMF)}

The MF model refers to a single type of unique relationship linking two types of entities. Let us consider a set $\left\{E_{1}, \ldots, E_{N}\right\}$ of $N$ types of entities connected by $M$ types of relations $\left\{R_{1}, \ldots, R_{M}\right\}$ which can be strongly correlated with each other. Let $W_{1}, W_{2}, \ldots, W_{n}(n \in N)$ be the latent factor matrices (designating the model parameters) of each of the entity types. These latent factors describe the entity and are constructed by considering each relationship to which the entity is associated. Taking into account the several relations of the domain, the objective function is given by [35] [24] [36]:

$$
O^{M R M F}=\sum_{r=1}^{M} \sum_{(s, i) \in R_{r}}\left(R_{r_{s i}}-w_{r 1_{s}} w_{r 2_{i}}^{T}\right)^{2}+\lambda\left(\sum_{n=1}^{N}\left\|W_{n}\right\|_{F}^{2}\right)
$$

With $R_{r}=\left\{\left(E_{1 r} ; E_{2 r}\right)\right\}(r=1 . . M)$.

When learning model parameters, each factor matrix is updated according to all the relationship types it implies until a common convergence is reached [8] or the maximum number of pre-defined iterations is achieved.

\section{Weighted Multi-Relational Matrix Factorization method (WMRMF)}

Assuming some relationships have more weight compared to others, a $\theta$ weight factor is added to the MRMF model. The objective function thus becomes [24] [36] [37]:

$o^{W M R M F}=\sum_{r=1}^{M} \Theta_{r} \sum_{(s, i) \in R_{r}}\left(R_{r_{s i}}-w_{r 1_{s}} w_{r 2_{i}}^{T}\right)^{2}+\lambda\left(\sum_{n=1}^{N}\left\|W_{n}\right\|_{F}^{2}\right)$

The weight function may be defined as follows:

$$
\Theta_{r}= \begin{cases}1, & \text { if } r \text { is the main relationship } \\ \theta, & \text { if }(0<\theta<1)\end{cases}
$$

For the learning process, the WMRMF model updates its latent factors using equations (7) and (9):

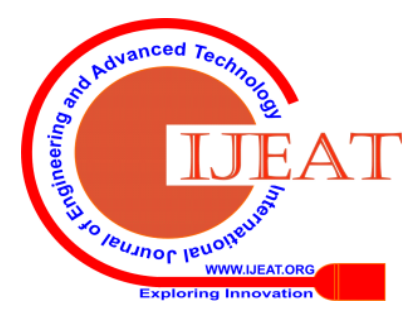


Multi-Relational and Social-Influence Model for Predicting Student Performance in Intelligent Tutoring Systems (ITS)

$$
\begin{aligned}
& w_{r 1_{s k}}^{\prime}=w_{r 1_{s k}}-\beta\left(\frac{\partial O^{\text {WMRMF }}}{\partial w_{r 1_{s k}}}\right) \\
& w_{r 2_{i k}}^{\prime}=w_{r 2_{i k}}-\beta\left(\frac{\partial O^{\text {WMRMF }}}{\partial w_{r 2_{i k}}}\right)
\end{aligned}
$$

With $\beta$ that is the learning rate. By determining the gradients $\frac{\partial O^{\text {WMRMF }}}{\partial w_{r 1_{s k}}}$ and $\frac{\partial O^{\text {WMRMF }}}{\partial w_{r 2_{i k}}}$, we finally obtain:

$$
\begin{gathered}
w_{r 1_{s k}}^{\prime}=w_{r 1_{s k}}+\beta\left(2 \Theta_{r} e_{r_{s i}} w_{r 2_{i k}}-\lambda w_{r 1_{s k}}\right) \\
w_{r 2_{i k}}^{\prime}=w_{r 2_{i k}}+\beta\left(2 \Theta_{r} e_{r_{s i}} w_{r 1_{s k}}-\lambda w_{r 2_{i k}}\right)
\end{gathered}
$$

\section{PROPOSED APPROACH}

We propose a multi-relational factorization approach that can integrate not only friendship relationships such as workgroup relationships, but also mutual influence values. The proposed methods are named So-MRMF (Multi-Relational Matrix Factorization and Social) and So-WMRMF (Weighted Multi-Relational Matrix Factorization and Social)

\section{A. Problem formulation}

Our approach is essentially based on the work done in [31]. We propose a multi-relational approach integrating workgroup relationships. In this approach, we consider the relationship "student-performs-task » as the main relationship. Figure 3 presents in the form of entity-association diagram, the information that we take into account in our model. At the completion of a task by the student, we associate a performance score and the number of indices requested by the student. To properly solve task, the student must know specific skills, and the task itself is also associated with the skills to be learned by the student. "Occurrence" attribute gives the number of times the student has learned the skill.

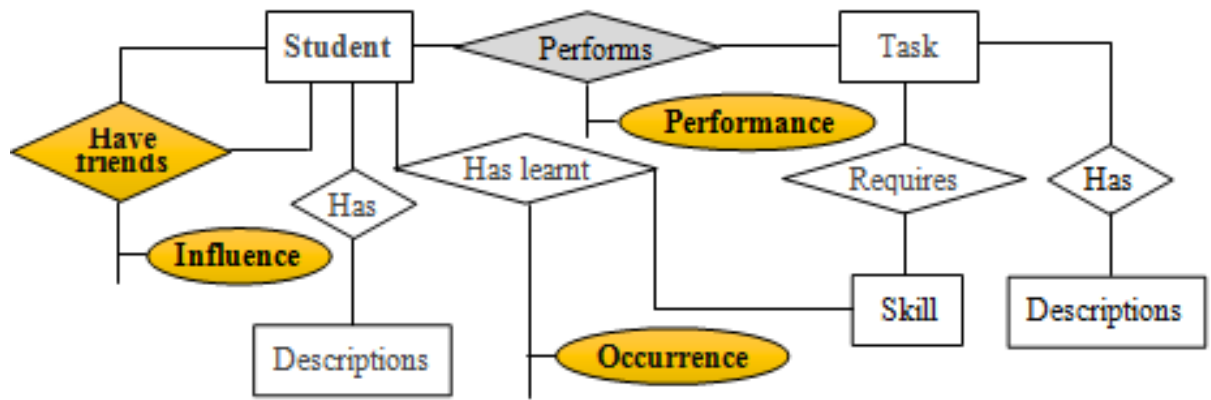

Fig. 3. Entity-association diagram for student performance prediction

\section{B. Relationship matrix with social influence effect}

In addition to the different relationships previously explored, our approach combines student friendly relationships such as workgroup relationships. The friendship network can essentially be modeled using a confidence graph of Figure 4 (a) or using a confidence matrix as shown in Figure 4 (b). In the confidence graph shown in Figure 4(a), 5 students (nodes, from $S_{1}$ to $S_{5}$ ) are connected to each other at 8 relationships (edges), and each relation is associated with a weight $T_{s, u}$ (influence value) in interval ] $\left.0 ; 1\right] . T_{s, u}$ is the influence value of student $u \in N_{s}$ ( $N_{s}$ being all student $s$ friends) on student $s$. Thus, the influence value of the student $S_{4}$ on student $S_{1}$ is equal to 0.2 while that of the student $S_{5}$ on the student $S_{1}$ is equal to 0.5 . For $S_{2}, S_{1}$ has more influence on his learning than $S_{4}$.

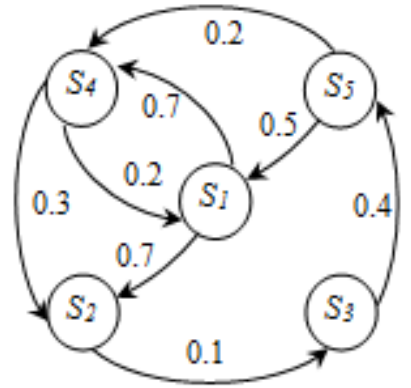

\begin{tabular}{|c|c|c|c|c|c|}
\hline$T$ & $S_{1}$ & $S_{2}$ & $S_{3}$ & $S_{4}$ & $S_{5}$ \\
\hline$S_{1}$ & 1 & & & 0.2 & 0.5 \\
\hline$S_{2}$ & 0.7 & 1 & & 0.3 & \\
\hline$S_{3}$ & & 0.1 & 1 & & \\
\hline$S_{4}$ & 0.4 & & & 1 & 0.2 \\
\hline$S_{5}$ & & & 0.4 & & 1 \\
\hline
\end{tabular}

\section{Fig. 4. Example of trust relationships}

Unlike the work done in [31], our matrix $T$ is not binary; the value $T_{s, n}$ has rather a social influence effect belonging to the interval $] 0 ; 1]$. The value 1 corresponds to the influence $T_{s, s}$ that a student $s$ has on himself. We hypothesize that the student has more impact on their own learning process than others have on him. This value taken is equal to 1 . An influence value $T_{s, n}=0$ means that there is no relationship of friendship between the student $s$ and the student $n$.

By integrating the fact that the behavior of a student $s$ is affected by his direct neighbors [31][38], likewise, $s$ characteristic vector depends on the characteristic vectors of its direct neighbors. This formulation is given by:

$$
\hat{W}_{1_{s}}=\frac{\sum_{u \in N_{s}} T_{s, u} w_{1_{u}}}{\sum_{u \in N_{s}} T_{s, u}}
$$


Where $\hat{w}_{1_{\mathrm{s}}}$ is the estimated characteristic vector (latent factor vectors) of student $s$, given the characteristic vectors of his direct neighbors. The graphical model of Figure 5 is an example representing the integration of the confidence matrix through latent factor matrix $W$.

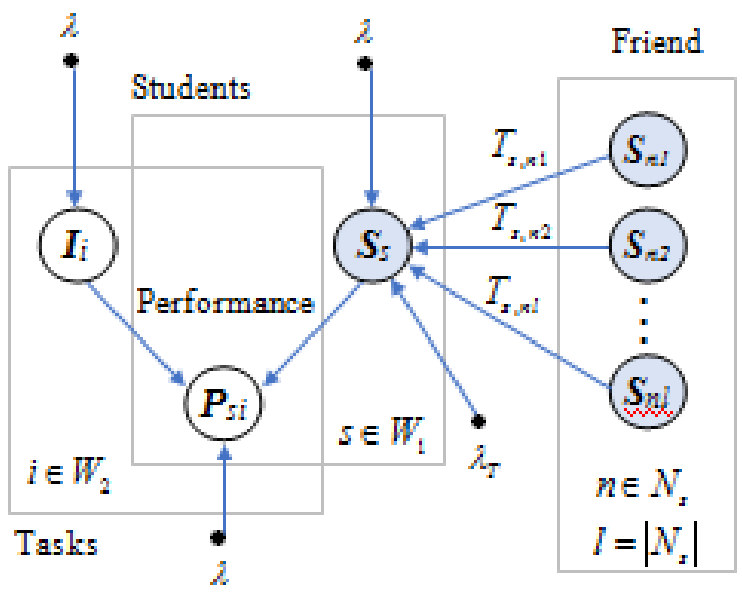

Fig. 5. Graphic model of performance prediction technique with social influence

$\lambda_{T}$ is a regularization term (regularization weight) that will be added to normalize objective function terms. By replacing $w_{1_{s}}$ by $\hat{w}_{1_{s}}$ in equation (1), for predicting student $s$ performance, we obtain:

$$
\hat{p}_{s i}=\sum_{f=1}^{F} \hat{w}_{1_{s f}} w_{2_{i f}}=\hat{w}_{1_{s}} w_{2_{i}}^{T}
$$

\section{Multi-Relational Matrix Factorization and Social-influence approach (So-MRMF)}

Taking into account the estimated characteristic vector of student $s$, the objective function expressed in equation (2) becomes:

$$
\begin{aligned}
& O^{S o-M F}=\sum_{(s, i) \in R}\left(R_{s i}-w_{1_{s}} w_{2_{i}}^{T}\right)^{2}+\lambda\left(\left\|W_{1}\right\|_{F}^{2}+\left\|W_{2}\right\|_{F}^{2}\right)+ \\
& \lambda_{T} \sum_{s=1}^{S}\left(w_{1_{s}}-\frac{\sum_{u \in N_{s}} T_{s, u} w_{1_{u}}}{\sum_{u \in N_{s}} T_{s, u}}\right)^{2}
\end{aligned}
$$

In this case, our multi-relational and social objective function is given by expression:

$$
O^{S O-M R M F}=O_{r=1}+O_{r \neq 1}
$$

So that functions $O_{r=1}$ and $O_{r \neq 1}$ are given by the equations (15) and (16)

$$
\begin{aligned}
& O_{r=1}=\sum_{(s, i) \in R}\left(R_{s i}-\hat{w}_{1 s} w_{2 i}^{T}\right)^{2}+\lambda\left(\left\|W_{1}\right\|_{F}^{2}+\left\|W_{2}\right\|_{F}^{2}\right)+ \\
& \lambda_{T} \sum_{s=1}^{S}\left(W_{1_{s}}-\frac{\sum_{u \in N_{s}} T_{s, u} W_{1_{u}}}{\sum_{u \in N_{s}} T_{s, u}}\right)^{2} \\
& O_{r \neq 1}=\sum_{r=2}^{M} \sum_{(s, i) \in R_{r}}\left(R_{r s i}-w_{r 1_{s}} w_{r 2_{i}}^{T}\right)^{2}+\lambda\left(\sum_{n=1}^{N}\left\|W_{n}\right\|_{F}^{2}\right)
\end{aligned}
$$

It is assumed that $r=1$ denotes the main relationship taking into account the entities "student" and "task". The objective function $O_{r=1}$ is thus defined for the value $r=1$. The objective function $O_{r \neq 1}$, for its part, is defined for any other value of $r(r \neq 1)$. The objective function (14) is optimized by using stochastic gradient descent. Thus, our So-MRMF model updates its parameters by equations (17) and (18) :

$$
\begin{gathered}
w_{r 1_{s k}}^{\prime}=\left\{\begin{array}{l}
w_{r 1_{s k}}+\beta\left(2 e_{s i} w_{r 2_{i k}}-\lambda w_{r 1_{s k}}\right)+\lambda_{T}(X+Y), \text { if } r=1 \\
w_{r 1_{s k}}+\beta\left(2 e_{r_{s i}} w_{r 2_{i k}}-\lambda w_{r 1_{s k}}\right), \text { if } r \neq 1
\end{array}\right. \\
w_{r 2_{i k}}^{\prime}=w_{r 2_{i k}}+\beta\left(2 e_{r_{s i}} w_{r 1_{s k}}-\lambda w_{r 2_{i k}}\right)
\end{gathered}
$$

$X$ and $Y$ expressions are given through equations (19) et (20)

$$
\begin{aligned}
& X=\left(\frac{T_{s, s}}{\sum_{u \in N_{s}} T_{s, u}}-1\right)\left(w_{1_{s k}}-\frac{\sum_{u \in N_{s}} T_{s, u} w_{1_{u k}}}{\sum_{u \in N_{s}} T_{s, u}}\right) \\
& Y=\sum_{t \in N_{S} \backslash s} \frac{T_{t, s}}{\sum_{w \in N_{t}} T_{t, w}}\left(w_{1_{t k}}-\frac{\sum_{w \in N_{t}} T_{t, w} w_{1_{w k}}}{\sum_{w \in N_{t}} T_{t, w}}\right)
\end{aligned}
$$

Equations (19) and (20) have been proposed to give a reduced form of equation (17).

\section{Weighted Multi-Relational Matrix Factorization and Social-influence approach (So-WMRMF)}

Considering the fact that some relations have more impact on the prediction than others, in weight term, the objective function of So-WMRMF model is given by:

$$
O^{S O-W M R M F}=O_{r=1}^{W}+O_{r \neq 1}^{W}
$$

Such that the functions $O_{r=1}^{W}$ and $O_{r \neq 1}^{W}$ are given by the equations (22) and (23)

$$
\begin{aligned}
& O_{r=1}^{W}=\Theta_{r} \sum_{(s, i) \in R}\left(R_{s i}-\hat{w}_{1_{s}} w_{2_{i}}^{T}\right)^{2}+\lambda\left(\left\|W_{1}\right\|_{F}^{2}+\left\|W_{2}\right\|_{F}^{2}\right)+ \\
& \lambda_{T} \sum_{s=1}^{S}\left(W_{1_{s}}-\frac{\sum_{u \in N_{s}} T_{s, u} w_{1_{u}}}{\sum_{u \in N_{s}} T_{s, u}}\right)^{2}
\end{aligned}
$$




$$
O_{r \neq 1}^{W}=\sum_{r=2}^{M} \Theta_{r} \sum_{(s, i) \in R_{r}}\left(R_{r_{s i}}-w_{r 1_{s}} w_{r 2_{i}}^{T}\right)^{2}+\lambda\left(\sum_{n=1}^{N}\left\|W_{n}\right\|_{F}^{2}\right)
$$

Our So-WMRMF model updates these parameters for each relationship via equations (24) and (25).

$$
w_{r 1_{s k}}^{\prime}=\left\{\begin{array}{l}
w_{r 1_{s k}}+\beta\left(2 \Theta_{r} e_{s i} w_{r 2_{i k}}-\lambda w_{r 1_{s k}}\right)+\lambda_{T}(X+Y), \text { if } r=1 \\
w_{r 1_{s k}}+\beta\left(2 \Theta_{r} e_{r_{s i}} w_{r 2_{i k}}-\lambda w_{r 1_{s k}}\right), \text { if } r \neq 1
\end{array}\right.
$$

$$
w_{r 2_{i k}}^{\prime}=w_{r 2_{i k}}+\beta\left(2 \Theta_{r} e_{r_{s i}} w_{r 1_{s k}}-\lambda w_{r 2_{i k}}\right)
$$

\section{E. Learning phase: proposal of an algorithm for updating parameters.}

The main issue of this technique is to find the optimal parameters $W_{1}, W_{2}, \ldots, W_{n}$, we proposed the algorithm 1 (using a stochastic gradient descent) iterative below to update our model parameters. The algorithm 1 proposed for the model So-WMRMF proceeds by initializing the parameters from the normal distribution $N\left(\mu, \sigma^{2}\right)$, taking for expectation $\mu=0$ and for standard deviation $\sigma=0.01$.

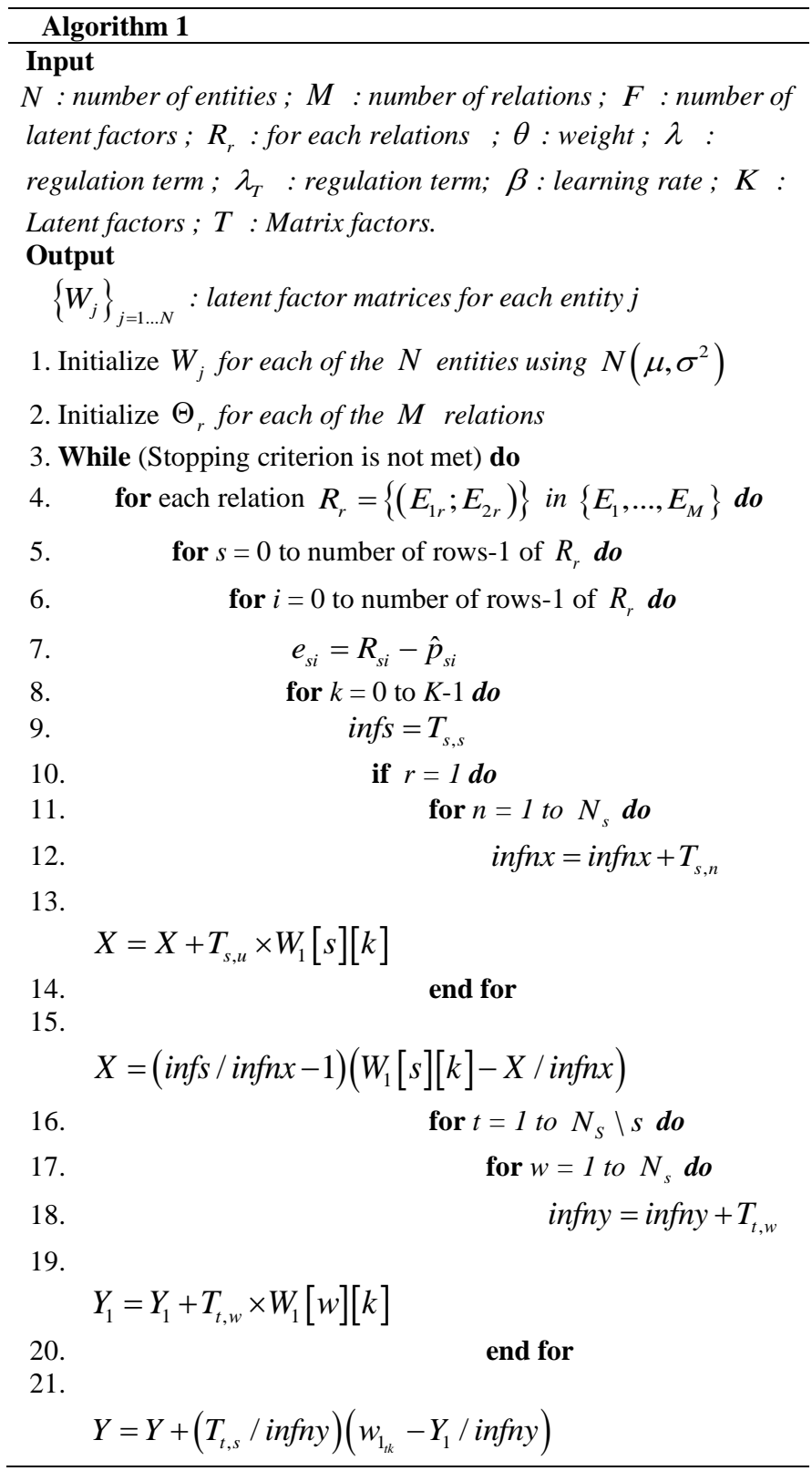

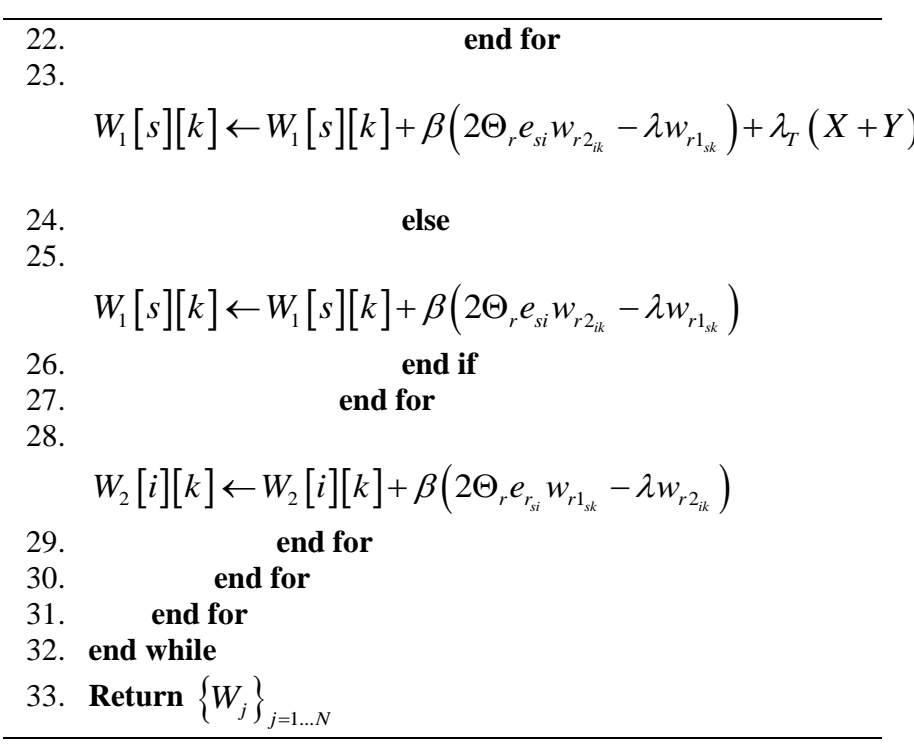

\section{EVALUATION OF THE PROPOSED MODELS}

\section{A. Data Set}

The data set for machine learning and testing comes from KDD Challenge 2010 ( Knowledge Discovery Data) database. This database is the result of interaction records between students and computer-assisted tutoring systems. In the KDD Challenge 2010 datasets ie "Algebra", the problem is the central element of the interaction between students and the Tutoring System. Students solve problems in the tutor and each interaction between the student and the system is recorded as a transaction line. The information about students, tasks and skills in this database is shown in Table 1.

Table 1. Information of students, tasks and performances

\begin{tabular}{|c|c|l|l|c|}
\hline Data set & \#Student & \#Task & \#Skill & \#Performance \\
\hline $\begin{array}{c}\text { Algebra } \\
\text { 2008-2009 }\end{array}$ & 3,310 & $1,422,200$ & 2,979 & $8,918,054$ \\
\hline
\end{tabular}

\section{B. Evaluation}

For our models' evaluation, we used the dataset "Algebra". Given the high data size of this database for our work environment, we reduced the sample for the evaluation. This sample is composed of:

- 247 students;

- 4000 tasks;

- 1664 skills;

In this sample, we extracted three (3) matrices. The first is related to the relationship "student-performs -tasks". This matrix is of dimension $247 \mathrm{x} 4000$. This matrix comprises 180676 known performances. We used two datasets: one for machine learning and one for testing. To perform cross validation, we used $9 / 10$ performance for the machine learning phase and 1/10 for the prediction phase. The second matrix relates to "student-as learnt-skill" relationship and the third to "task-requires-skill" relationship. Since the learning base does not have student group relationships, we simulated two group formation scenarios in all of the 247 students used for experiment. For each of the two scenarios, 40 groups of 4 students are formed. 160 students belong to a group while 87 belong to no group. 
In the scenario 1 noted So1., The influence values between students are all set at 1 . In the scenario 2 noted So2., these values are a function of the performances obtained by each student in the group. The average yield of a member of the group is calculated, in the interval ] 0;0.5], on the basis of the tasks performed. This yield is taken by default as the influence value of this member on the others. However, the influence value of a member of the group on itself is equal to 1.

\section{Results}

We implement So-MF, So-MRMF and So-WMRMF models taking into account three attributes (three relations) and the group relation as described in our association entity diagram. Our work environment is a 64-bit operating system computer, 4GB of ream, intel Core i3. Our model is designed in Python language.

To compare our approach with classical matrix factorization methods (MF, MRMF and WMRMF), we used the RMSE (Root Mean Squared Error) metric. This metric is calculated as follows :

$$
R M S E=\sqrt{\frac{\sum_{(r, s, i) \in D^{\text {test }}}\left(p_{s i}-\hat{p}_{s i}\right)^{2}}{\left|D^{\text {test }}\right|}}
$$

For this, the parameters used to optimize the model are contained in table 2.

Table 2. Optimizing parameters

\begin{tabular}{|c|c|}
\hline Methods & Parameter \\
\hline$M F$ & $\begin{array}{l}K=3 ; \# \text { iter }=30 ; \beta=2.10^{-3} ; \\
\lambda=55.10^{-5}\end{array}$ \\
\hline $\begin{array}{l}\text { So1-MF } \\
\text { So2-MF }\end{array}$ & $\begin{array}{l}K=3 ; \text { \#iter }=60 ; \beta=2.10^{-3} ; \\
\lambda=15.10^{-5} ; \lambda_{T}=15.10^{-5}\end{array}$ \\
\hline $\begin{array}{l}\text { MRMF ; } \\
\text { So1-MRMF ; } \\
\text { So2-MRMF ; }\end{array}$ & $\begin{array}{l}K=4 ; \# \text { iter }=70 ; \beta=2.10^{-3} ; \\
\lambda=55.10^{-5} ; \lambda_{T}=55.10^{-5}\end{array}$ \\
\hline $\begin{array}{l}\text { WMRMF ; } \\
\text { So1-WMRMF ; } \\
\text { So2-WMRMF ; }\end{array}$ & $\begin{array}{l}K=4 ; \# \text { iter }=80 ; \beta=2.10^{-3} ; \\
\lambda=55.10^{-5} ; \lambda_{T}=55.10^{-5} ; \\
\theta \in\{0.85 ; 0.80 ; 0.70\}\end{array}$ \\
\hline
\end{tabular}

Figure 6 gives the results of the experiments carried out by applying the two scenarios described above to each of the MF, MRMF and WMRMF methods.

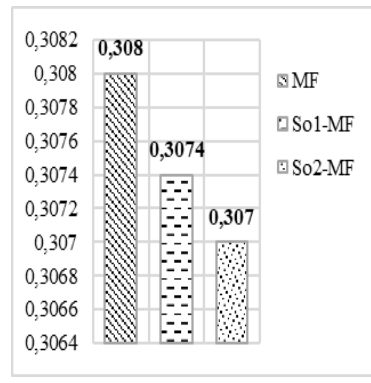

(a) RMSE with MF method

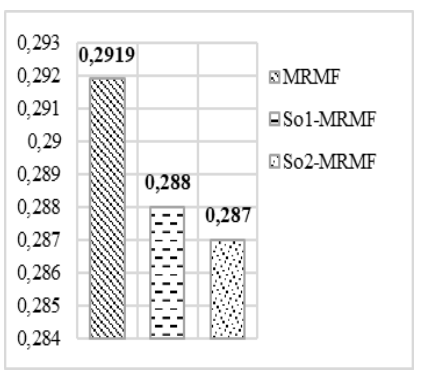

(b) RMSE with MRMF method

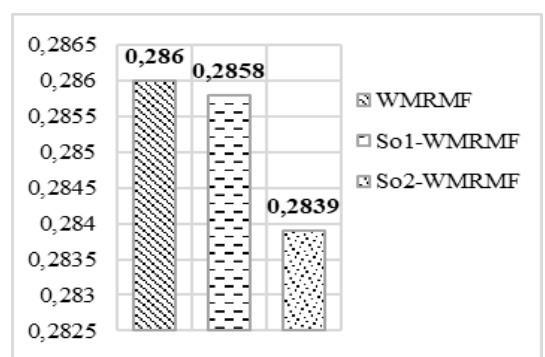

(c) RMSE with WMRMF method

Fig. 6. RMSE results on Algebra data set using the two scenarios So1 and So2

In Figure 6 (a), So1-MF and So2-MF models outperform the MF model. Figure 6 (b) shows that So1-MRMF and So2-MRMF models are significantly more accurate than the MRMF model. In Figure 6 (c), the So1-WMRMF model is significantly better compared to the WMRMF model while the So2-WMRMF model greatly outperforms WMRMF and So1-WMRMF models. Figure 7 gives a summary of RMSE metric for So2-MF, So2-MRMF and So2-WMRMF methods.

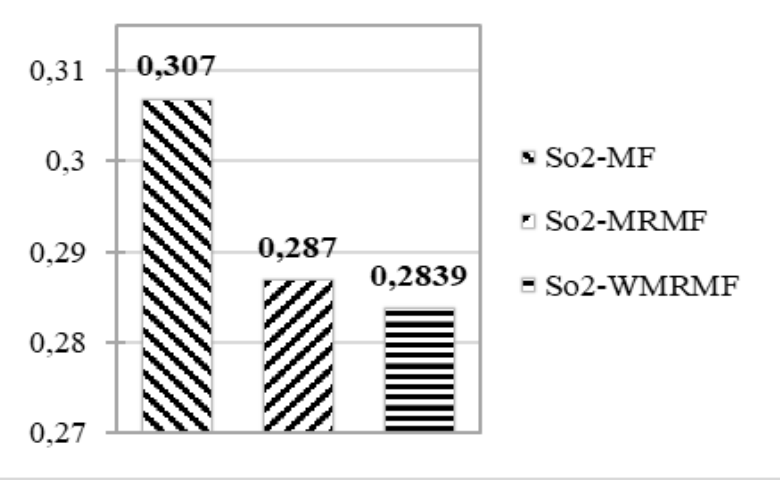

Fig. 7. RMSE results on Algebra data set using the two scenarios So1 and So2

These results show that the So2-WMRMF model outperforms all other models. Taking into account the influence factor on Weighted Multi-Relational Matrix Factorization, in order to predict performance, gives not only a better accuracy of prediction on the performances, but also, that it presents better results compared to the methods of standard Matrix Factorization.

\section{CONCLUSION}

In this paper, we have proposed a student performance prediction approach that integrates not only friendship relationships such as workgroup relationships, but also mutual influence values into Weighted Multi-Relational Matrix Factorization method. By applying RMSE metric to our models (So2-MF, So2-MRMF, So2-WMRMF), experimental results from the KDD Challenge 2010 database have shown that this approach can refine the accuracy of student performance prediction. The So2-WMRMF model outperforms all other models. In future work, the addition of some information such as the influence factor per student and per task and the definition of an influence value that takes advantage of real-life situations, would further improve the results of students' performances predictions in ITS. 


\section{REFERENCES}

1. R. J. Seidel and O.-C. Park, "An Historical Perspective and a Model for Evaluation of Intelligent Tutoring Systems," vol. 10 issue: 2, pp. 103-128, 1 Mars 1994

2. B. P. Woolf, "Building Intelligent Interactive Tutors, Student-Centered Strategies for Revolutionizing E-Learning," 2008.

3. W. J. Hawkins, N. T. Heffernan and R. S. Baker, "Learning Bayesian Knowledge Tracing Parameters with a Knowledge Heuristic and Empirical Probabilities," in International Conference on Intelligent Tutoring Systems, 2014.

4. J. Self, "The role of student models in learning environments," in Transactions of the Institute of Electronics, Information and Communication Engineers, 1994.

5. I. Magnisalis, S. Demetriadis and A. Karakostas, "Adaptive and Intelligent Systems for Collaborative Learning Support: A Review of the Field," IEEE Transactions on Learning Technologies, vol. 4, pp. 5-20, 2011.

6. R. Lukašenko, "Development of Student Model for Support of Intelligent Tutoring System Functions," RTU Research Information System, 2012.

7. A. T. Corbett and J. R. Anderson, "Knowledge Tracing: Modeling the Acquisition of Procedural Knowledge," Kluwer Academic Publishers, pp. 253-278, 1995.

8. M. Feng, N. Heffernan and K. Koedinger, "Addressing the assessment challenge with an online system that tutors as it assesses," User Modeling and User-Adapted Interaction, p. 243-266, 2009.

9. H. Cen, K. Koedinger and B. Junker, "Learning factors analysis a general method for cognitive model evaluation and improvement," Intelligent Tutoring Systems, vol. 4053, p. 164-175, 2006.

10. I. A. A. Amra and A. . Y. A. Maghari, "Students Performance Prediction Using KNN and Naïve Bayesian," in 8th International Conference on Information Technology (ICIT), 2017.

11. K. B. Eashwar, R. Venkatesan and D. Ganesh, "Student performance prediction using SVM," International Journal of Mechanical Engineering and Technology. 8., vol. 8, pp. 649-662, 2017.

12. H. Al-Shehri, A. Al-Qarni, L. Al-Saati, A. Batoaq, H. Badukhen, S. Alrashed, J. Alhiyafi and S. O. Olatunji , "Student Performance Prediction Using Support Vector Machine and K-Nearest Neighbor," in IEEE 30th Canadian Conference on Electrical and Computer Engineering (CCECE), 2017.

13. K. B. Eashwar, R. Venkatesan and D. Ganesh, "Student performance prediction using svm," International Journal of Mechanical Engineering and Technology (IJMET), 2017.

14. H. Lu and J. Yuan, "Student Performance Prediction Model Based on Discriminative Feature Selection," 2018.

15. H. Hamsa, S. Indiradevi and J. Kizhakkethottam, "Student Academic Performance Prediction Model Using Decision Tree and Fuzzy Genetic Algorithm," in Global Colloquium in Recent Advancement and Effectual Researches in Engineering, Science and Technology, 2016.

16. R. Hasan, S. Palaniappan, A. R. e. A. Raziff, S. Mahmood and K. U. Sarker, "Student Academic Performance Prediction by using Decision Tree Algorithm," in 4th International Conference on Computer and Information Sciences (ICCOINS), 2018.

17. F. Okubo, T. Yamashita, A. Shimada and H. Ogata, "A Neural Network Approach for Students' Performance Prediction," in the Seventh International Learning Analytics \& Knowledge Conference, 2017.

18. R. Ulloa-Cazarez and C. Martín, "Neural Networks for predicting student performance in online education," vol. 16, no. 7, July 2018.

19. N. Thai-Nghe, L. Drumond, A. Krohn-Grimberghe and L. Schmidt-Thieme, "Recommender System for Predicting Student Performance," in 1st Workshop on Recommender Systems for Technology Enhanced Learning, 2010.

20. E. Jembere, R. Rawatlal and A. W. Pillay, "Matrix Factorisation for Predicting Student Performance," in 7th World Engineering Education Forum (WEEF), 2017.

21. E. Jembere, R. Rawatlal and A. W. Pillay, "Matrix Factorisation for Predicting Student Performance," in 7th World Engineering Education Forum (WEEF), 2017.

22. N. Thai-Nghe, T. Horvath and L. Schmidt-Thieme, "Factorization Models for Forecasting Student Performance," 2011.

23. N. Thai-Nghe, L. Drumond, T. Horváth, A. Krohn-Grimberghe, A. Nanopoulos and L. Schmidt-Thieme, "Factorization Techniques for Predicting Student Performance," 2011.

24. N. Thai-Nghe and L. Schmidt-Thieme, "Multi-Relational Factorization Models for Student Modeling in Intelligent Tutoring Systems," in Seventh International Conference on Knowledge and Systems Engineering, 2015.
25. P. Nedungadi and T. K. Smruthy, "Personalized Multi-relational Matrix Factorization Model for Predicting Student Performance," 2016.

26. L. Springer, M. E. Stanne and S. S. Donovan, "Effects of small-group learning in science, mathematics, engineering, and technology: A meta-analysis. Review of Educational Research," pp. 21-51, 1999.

27. D. W. Johnson, R. T. Johnson and K. A. Smith, "Cooperative learning: Improving university instruction by basing practice on validated theory," Journal on Excellence in College Teaching, p. 85-118, 2014.

28. H. Ma, H. Yang, M. R. Lyu and I. King, "Social Recommendation Using Probabilistic Matrix Factorization," CIKM, p. 931-940, 2008.

29. H. Ma, I. King and M. R. Lyu, "Learning to recommend with social trust ensemble," in Proceedings of the 32nd international ACM SIGIR conference on Research and development in information retrieval, 2009.

30. M. Jamali and M. Ester, "A Matrix Factorization Technique with Trust Propagation for Recommendation in Social Networks," RecSys2010, p. 26-30, 2010.

31. H.-L. Thanh-Nhan, L. Huy-Thap and N. Thai-Nghe, "Toward integrating social networks into intelligent tutoring systems," in 9th International Conference on Knowledge and Systems Engineering (KSE), 2017.

32. J. Bicans and J. Grundspenkis, "Student Learning Style Extraction from On-Campus Learning Context Data," 2017.

33. L. Chen, G. Chen and F. Wang, "Recommender systems based on user reviews : the state of the art," User Modeling and User-Adapted, vol. 25, no. 2, p. 99-154, 2015.

34. G. Takacs, I. Pilaszy and B. Nemeth, "On the Gravity Recommendation System," Proceedings of KDD Cup and Workshop, 2007.

35. C. Lippert, S. H. Weber, Y. Huang and V. Tresp, "Relation Prediction in Multi-Relational Domains using Matrix Factorization," in NIPS 2008 Workshop on Structured Input - Structured Output., 2008.

36. "An Approach for Multi-Relational Data Context in Recommender Systems," in Asian Conference on Intelligent Information and Database Systems, 2017.

37. H. Li, X. Diao, J. Cao and Q. Zheng, "Collaborative filtering recommendation based on all-weighted matrix factorization and fast optimization_2018," Ieee Access, 2018.

38. M. Jamali and M. Ester, "A Matrix Factorization Technique with Trust Propagation for Recommendation in Social Networks," 2010.

\section{AUTHORS PROFILE}

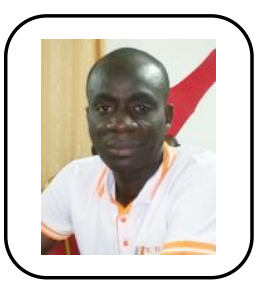

Kouamé Abel ASSIELOU received the master's degree in Computer Engineering from the University Nangui Abrogoua (Côte d'Ivoire) and also the diploma of Engineering in network and telecommunication at the training institute for high expertise of BNETD (Bureau National d'étude technique et de Développement).

He is currently a PhD student at the INP-HB (Institut National Polytechnique Félix Houphouët Boigny de Yamoussoukro). His current research interest is related to designing, modeling of Intelligent Tutoring Systems (ITS).

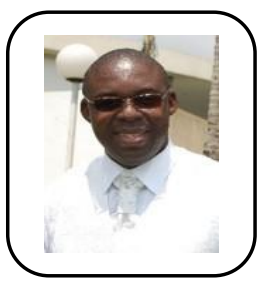

Théodore Cissé HABA received the Ph.D. degree from the University of Paul Sabatier de Toulouse III (France). He is currently a Professor and researcher at Institut National Polytechnique Houphouët Boigny (INP-HB) of Yamoussoukro (Côte d'Ivoire). He worked on the frequency behavior of fractional impedance microelectronic devices. Currently, his is working on digital processing and the implementation of electronic

devices for solar. 


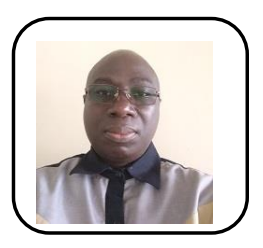

Dr Lambert Tanon KADJO received his BS in Computer Science from University of Cocody (Côte d'Ivoire) in 2003, his M.S. in Numerical Analysis from University of Abobo-Adjamé Abidjan in Côte d'Ivoire in 2005 and professional Master of engineering from CFTIC (Centre de Formation en Technologies de l'Information et de la Communication) at INPHB - Abidjan in Cote d'Ivoire in 2007. He received $\mathrm{PhD}$ in Telecommunications at University of Cheikh Anta Diop of Dakar in Senegal in 2009.

His research interests include IPv6 Mobility, IP Multicast, IP Network security, 4G networks, Social network. He is authors/co-authors of 11 papers published in these arears. He is working as Assistant Professor at Institut National Polytechnique Felix HOUPHOUET Boigny (INP-HB). profile which contains their education details, their publications, research work, membership, achievements, with photo that will be maximum 200-400 words.

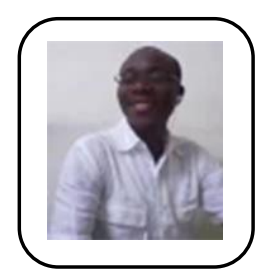

YAO k. Daniel received the Ph.D. degree in psychology from the University of Félix Houphouët Boigny (Abidjan/Ivory Coast). He is an Assistant Professor in the university jean Lorougnon Guédé de Daloa. (Côte d'Ivoire). He conducted a series of works on educational psychology, urban violence and juvenile marginality.

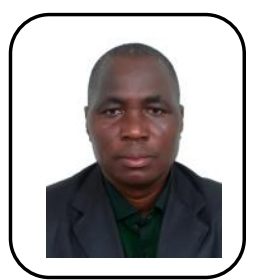

Bi Tra GOORE He is currently a Professor with the INP-HB (Institut National Polytechnique Houphouët Boigny de Yamoussoukro). Where he spent several years in teaching the computer science and Network. He is a member of the rearch laboratory LARIT ( Laboratoire de recherche en Informatique et Télécommunication). His research field include design, modeling, and performance evaluation of wireless sensor networks design, His current research interests include design, modeling, and performance evaluation of networks and telecommunication system 